Como citar este artículo: López-López E, López-Carbajal MJ, Ortiz AA. Caracterización clínica de los pacientes con diabetes mellitus del registro estatal de diabetes en un Estado de México. Revista Biosalud 2015; 14(1): 51-56. DOI: 10.17151/biosa.2015.14.1.6

\title{
CARACTERIZACIÓN CLÍNICA DE LOS PACIENTES CON DIABETES MELLITUS DEL REGISTRO ESTATAL DE DIABETES EN UN ESTADO DE MÉXICO
}

\author{
Erika López-López ${ }^{1}$ \\ Mario Joaquín López-Carbajal² \\ Alicia Armida Ortiz Gress ${ }^{3}$
}

\section{RESUMEN}

Introducción: La diabetes mellitus representa un reto para los sistemas de salud debido a la complejidad de la atención, la calidad y el acceso a tratamientos. Objetivo: Describir las características clínicas de los pacientes con diabetes mellitus del registro estatal de diabetes del Estado de Hidalgo en México de los años 2007 a 2012. Material y Métodos: Estudio transversal descriptivo del Registro Estatal de Diabetes (RED) durante 2007 al 2012. Se realizó descripción estadística simple para caracterizar a la población del estudio, proporciones y razones para variables cualitativas y para variables cuantitativas medidas de tendencia central y de dispersión (media, mediana, moda, desviación estándar y varianza). Para el análisis de las variables continuas se hizo la prueba de $t$ de Student. Resultados: Se identificaron 10.198 pacientes del RED, el 66,4\% fueron mujeres y el 33,52\% hombres. La media de edad fue 52,2 $\pm 12,8$ años. Respecto a la glucosa en ayuno, la media fue de $239,1 \pm 95,3 \mathrm{mg} / \mathrm{dl}$; la media de glucosa postprandial $293,6 \pm 122,7 \mathrm{mg} / \mathrm{dl} \mathrm{y}$ la de glucosa casual $247,9 \pm 102,3 \mathrm{mg} / \mathrm{dl}$. Las actividades del RED incluyeron a 5.795 personas que fueron detectadas en ese momento, lo que corresponde al 56,8\%. De las cuales, 11,9\% manifestó tener visión borrosa, $48,5 \%$ poliuria, $4,7 \%$ infecciones, $44,07 \%$ polidipsia, $13,19 \%$ polifagia, $26,9 \%$ pérdida de peso y $35,2 \%$ fatiga.
Al comparar las que estaban en tratamiento con las que ingresaban no encontramos diferencias estadísticas. Mientras que las que se encontraban en tratamiento, quienes mencionaron solo tener dieta fueron el 33,5\%, metformina $72,58 \%$, sulfonilureas $62,9 \%$, acarbosa 3,59\%, insulina 9,4\%, rosiglitazona 0,66\%. Discusión: El RED tiene estándares de control glicémico bajo. Se sugiere la elaboración de indicadores internos para que estos les permitan realizar la toma de decisiones desde el interior de la unidad y ofrecer una mejor calidad de atención, el cumplimiento y oportunidad.

Palabras clave: evaluación de programas, diabetes mellitus, calidad, México.

\section{CLINICAL CHARACTERIZATION OF PATIENTS WITH DIABETES MELLITUS FROM THE STATE REGISTER OF DIABETES IN A STATE OF MEXICO}

\section{ABSTRACT}

Introduction: Diabetes mellitus is a challenge for health systems due to the complexity of care, and the quality and access to treatment. Objective: To describe the clinical characteristics of patients in the Diabetes Mellitus State Register in the state of Hidalgo in Mexico from 2007 to 2012.

\footnotetext{
${ }^{1}$ Maestría en Ciencias de la Salud. Dirección de Investigación en Salud de los Servicios de Salud de Hidalgo. Hidalgo, México. Correo electrónico: erika.lopez.83@hotmail.com

2 Médico especialista en epidemiología. Dirección de Investigación en Salud de los Servicios de Salud de Hidalgo. Hidalgo, México. Correo electrónico: epiblasto@yahoo.com.mx

${ }_{3}$ Trabajadora Social. Dirección de Investigación en Salud de los Servicios de Salud de Hidalgo. Hidalgo, México. Correo electrónico: alicagress@gmail.com
} 
Methods: Cross-sectional descriptive study of the Diabetes State Register (DSR) between 2007 and 2012. Simple statistics description to characterize the studied population, proportions and reasons for qualitative variables and for quantitative measures of central tendency and continuous and dispersion variables (media, median, mode, deviation, standard and variance) were carried out. The $t$ student test was used for the analysis of continuous variables. Results: 10,198 patients from the Diabetes State Register were identified; $66.4 \%$ were women and $33.52 \%$ men. The mean age was $52.2 \pm 12.8$. Regarding fasting glucose, the mean was $239.1 \pm 95.3 \mathrm{mg} / \mathrm{dl}$; the post-prandial glucose mean was $293.6 \pm 122.7$ $\mathrm{mg} / \mathrm{dl}$ and casual glucose was $247.9 \pm 102.3 \mathrm{mg} /$ dl. The DSR activities included 5,795 people that were detected at that time which correspond to $56.8 \%$ from which $11.9 \%$ reported having

\section{INTRODUCCIÓN}

La Diabetes Mellitus (DM) comprende un grupo de enfermedades caracterizadas por hiperglucemia. Se clasifica en tipo 1 cuando hay defectos en la secreción de insulina y tipo 2 cuando hay defectos en la acción de la insulina. Es una de las principales causas de ceguera, insuficiencia renal, amputaciones no traumáticas, forma parte de las 10 causas más frecuentes de hospitalización en adultos (1), y representa más del $85 \%$ de la DM en los países en desarrollo $(2,3)$. Asimismo, representa un reto para los sistemas de salud debido a la complejidad de la atención, la calidad y el acceso a tratamientos (4).

En México, la Encuesta Nacional de Salud y Nutrición 2012 (ENSANUT 2012) identificó a 6,4 millones de adultos mexicanos con DM y se estima podría ser incluso el doble, de acuerdo a la evidencia previa sobre el porcentaje de personas que desconocían su condición (5). En 2012, en comparación con los resultados nacionales, la prevalencia de diagnóstico médico blurred vision, polyuria $48.5 \%$, infections $4.7 \%$, polydipsia $44.07 \%$, polyphagia $13.19 \%$, weight loss $26.9 \%$ and fatigue $35.2 \%$. When comparing people under treatment with those admitted, no statistical difference was found While those who were under treatment, who mentioned only having diet were $33.5 \%$, metformin $72.58 \%$, sulfonylureas $62.9 \%$, acarbose $3.59 \%$, insulin $9.4 \%$, and rosiglitazone $0.66 \%$. Discussion: The Diabetes State Register has low glycemic control standards. Development of internal indicators is suggested to allow medical staff to make decisions from inside the unit and provide a better quality of care, compliance and opportunity.

Key words: record, diabetes mellitus, quality, Mexico.

previo de diabetes en Hidalgo fue de $8,2 \%$, se ubicó debajo de la media nacional $(9,17 \%)(6)$.

En México se han desarrollado diferentes registros, tal es el caso del Sistema Nacional de Vigilancia Epidemiológica, donde se registra la morbilidad de la diabetes dentro del Sistema Único de Información Epidemiológica (SUIVE), que incluye la notificación de DM (CIE10 E10-E14); la mortalidad es registrada y analizada a través del Sistema Epidemiológico y Estadístico de Defunciones. Estos sistemas permiten ubicar a esta enfermedad como uno de los problemas prioritarios en la salud pública del país (7).

En el año 2005, se diseñó un Registro Estatal de Diabetes (RED) con el fin de contar con estadísticas locales que permitieran mejorar la calidad de la atención e identificar las condiciones clínicas de los pacientes (8). Dicho registro incluye información como: escolaridad, ocupación, edad, domicilio, tipo de diabetes, condiciones clínicas de diagnóstico, cifra de glucosa inicial y actual, control metabólico; tratamiento previo, complicaciones agudas, 
factores de riesgo cardiovascular, complicaciones crónicas y atención recibida.

El RED comprende a los pacientes de primer nivel de atención, de los 474 centros de salud en todo el Estado de Hidalgo, tuvieran o no seguridad social, y sin considerar su estado de salud. Asimismo, el RED está incluido dentro del Seguro Popular (9) el cual cuenta con un Catálogo Único de Servicios de Salud (CAUSES), donde se encuentra un listado con las intervenciones en salud que son gratuitas (10). Por ello, el objetivo del estudio fue describir las características clínicas de los pacientes con diabetes mellitus del RED de los años 2007 a 2012

\section{MATERIAL Y MÉTODO}

El diseño del estudio es transversal, descriptivo del RED. Durante el segundo trimestre de 2013, personal de la Coordinación de investigación capturó 11.948 encuestas correspondientes al RED de 2007 al 2013. Este instrumento incluyó aspectos sociales, clínicos y paraclínicos. Los criterios clínicos fueron de acuerdo a los establecidos por la "NORMA Oficial Mexicana NOM-015-SSA2-2010, Para la prevención, tratamiento y control de la diabetes mellitus" (11) y la guía de práctica clínica para el tratamiento de la diabetes mellitus tipo 2 en el primer nivel de atención (12).
Se realizó descripción estadística simple para caracterizar a la población del estudio, proporciones y razones para variables cualitativas y para variables cuantitativas medidas de tendencia central y de dispersión (media, mediana, moda, desviación estándar y varianza). Para el análisis de las variables continuas se hizo la prueba de $t$ de Student.

\section{Consideraciones éticas}

Este trabajo fue sometido a la evaluación de los comités de ética e investigación de la Secretaría de Salud, según lo señalado en el Reglamento de la Ley General de Salud en materia de investigación, este estudio corresponde a una investigación sin riesgo ya que el análisis será a partir de una base de datos (13).

\section{RESULTADOS}

Se identificaron 10.198 pacientes del RED, el $66,4 \%$ fueron mujeres y el 33,52\% hombres. La media de edad fue 52,2 $\pm 12,8$ años. En el momento en que se diagnosticó DM, se encontraron los valores de la Tabla 1. Las actividades del RED incluyeron a 5.795 personas que fueron detectadas en ese momento, lo que corresponde al 56,8\%. De las cuales, 11,9\% manifestó tener visión borrosa, $48,5 \%$ poliuria, $4,7 \%$ infecciones, $44,07 \%$ polidipsia, $13,19 \%$ polifagia, $26,9 \%$ pérdida de peso y $35,2 \%$ fatiga.

Tabla 1. Valores de glucosa en el momento del diagnóstico.

\begin{tabular}{ccccc}
\hline CONDICIÓN INICIAL & Frecuencia & Promedio & (Mín-Máx) & Desviación estándar \\
\hline Glucosa en ayuno & 8670 & 239,08 & $(43-946)$ & 95,29 \\
Glucosa postprandial & 371 & 293,61 & $(101-800)$ & 122,86 \\
Glucosa casual & 746 & 247,85 & $(74-734)$ & 102,30 \\
\hline
\end{tabular}

Mientras que las que se encontraban en tratamiento (Tabla 2), quienes mencionaron solo tener dieta fueron el $33,5 \%$, metformina $72,58 \%$, sulfonilureas $62,9 \%$, acarbosa $3,59 \%$, insulina $9,4 \%$, rosiglitazona $0,66 \%$. 
Tabla 2. Valores de glucosa en pacientes en tratamiento.

\begin{tabular}{ccccc}
\hline $\begin{array}{c}\text { TRATAMIENTO } \\
\text { ACTUAL }\end{array}$ & Frecuencia & Promedio & (Mín-Máx) & Desviación estándar \\
\hline Glucemia capilar & 7717 & 197,13 & $(48-808)$ & 87,16 \\
Glucemia venosa & 2788 & 210,32 & $(52-940)$ & 85,95 \\
Hemoglobina glucosilada & 596 & 8,85 & $(0,1-16,9)$ & 2,57 \\
\hline
\end{tabular}

Respecto a obesidad el 32,7\% fue positivo y $2,29 \%$ reportó tabaquismo. En relación a las glucosurias, solo 729 personas se realizaron el estudio y el $42,94 \%$ fue positivo, cetonurias fueron 607 personas y positivas el 13,01\%, microalbuminuria se realizó el estudio 304 personas y positivas fueron $5,92 \%$. De acuerdo a las complicaciones, $0,5 \%$ ya tenía una amputación, $2,47 \%$ neuropatía, $1,33 \%$ nefropatía, $2,08 \%$ retinopatía, $0,92 \%$ pie diabético. Finalmente, la enfermedad coronaria se presentó en un $0,2 \%$, ceguera $0,54 \%$, enfermedad cardiovascular $0,37 \%$. Cabe mencionar que el 10,9\% reportó que el médico que la atendió le revisó el fondo de ojo y solo el 7,28 tuvo una revisión de pies por su médico tratante.

\section{DISCUSIÓN}

Infortunadamente, el RED muestra que los estándares de control glicémico se encuentran por debajo de la meta de control metabólico que sugieren las guías clínicas. Es preocupante que no existan diferencias estadísticamente significativas entre las personas que ingresan por primera vez al RED y las que se encuentran en tratamiento, pues la evolución natural de la diabetes puede modificarse con acciones que cambien el curso clínico de las condiciones que determinan su incidencia (14). Estudios en Estados Unidos de América (EUA) reportan que solo $37 \%$ de los individuos cumplen con las recomendaciones (15). En tanto, el monitoreo de la glucemia se presentó elevado en comparación con el 19,5\% reportado en Colombia (16). Mientras que en México, de acuerdo a la
ENSANUT 2012, $80 \%$ de los pacientes con diabetes reciben tratamiento farmacológico. Sin embargo, hasta el 30\% logra llegar a metas de control. Esta complejidad del tratamiento de la enfermedad contribuye a la falta de eficacia terapéutica en todo el mundo (17).

El RED mostró debilidad en la atención del pie diabético, lo que impacta en que el $0,5 \%$ de los usuarios que ingresaron ya presentan amputación en alguno de los miembros inferiores. Estos casos son similares al 6,1\% descrito por Reid et al. entre aborígenes canadienses (18). Lo anterior es relevante ya que $75 \%$ de quienes tienen úlceras de pie diabético son varones, lo cual representa $18 \%$ de las visitas a las salas de emergencia, y no existe una educación por el uso de calzado adecuado (15).

La falta de monitoreo del funcionamiento renal del RED, puede pasar por alto la ventana de oportunidad que brinda la detección oportuna (19) y disminuir el impacto socioeconómico de la terapia renal sustitutiva (20). Laliberté et al., reportaron que $14,0 \%$ de quienes tienen DM e hipertensión desarrollan insuficiencia renal crónica en un periodo de seis años, y su costo anual en los servicios de salud aumenta 3,8 veces (21). Mientras que Barquera et al., muestran que el promedio de costos en diabetes durante un año (sin complicaciones) es equivalente a US $\$ 707$, y cuando las complicaciones aparecen este costo incrementa $75 \%$ (22).

Debido a que los usuarios del RED cuentan con un Catálogo Único de Servicios de Salud (que brinda el Seguro Popular), muchos de 
los estudios no están incluidos de manera gratuita. Esto implica que enfrentan gastos de bolsillo cuando necesitan procedimientos y medicamentos, como la utilización de exámenes de hemoglobina glucosilada. Ello puede explicar que solo 596 personas accedieron a realizarse esta prueba.

Las limitaciones del estudio son las inherentes al utilizar base de datos secundarias, inicialmente porque los datos utilizados son los registros electrónicos creados por médicos, que pueden dar lugar a la grabación de lo que deben hacer en lugar de lo que realmente hacen durante una consulta.

\section{CONCLUSIONES}

La vigilancia epidemiológica en México ha permitido identificar la magnitud y efecto tanto en la morbilidad como en la mortalidad de la diabetes. Este registro permitió identificar las condiciones clínicas del paciente con DM. Las condiciones son desalentadoras ya que la oportunidad de diagnóstico está desaprovechada y los resultados de los tratamientos actuales no muestran efectividad en el tratamiento. Se sugiere la elaboración de indicadores internos para que estos les permitan realizar la toma de decisiones desde el interior de la unidad y ofrecer una mejor calidad de atención, el cumplimiento y oportunidad.

\section{REFERENCIAS}

1. Rull JA, Aguilar-Salinas CA, Rojas R, Ríos-Torres JM, Gómez-Pérez FJ, Olaiz G. Epidemiology of type 2 diabetes in Mexico. Arch Med Res 2005; 36:188-196.

2. International Diabetes Federation. Diabetes atlas. 3rd edition. Bélgica: International Diabetes Federation; 2008.

3. Expert Committee on the Diagnosis and Classification of Diabetes Mellitus. Report of the Expert Committee on the Diagnosis and Classification of Diabetes Mellitus. Care 1997; 20:1183-1197.

4. Genuth S, Alberti KG, Bennett P, Buse J, Defronzo R, Kahn R, et al. Expert Committee on the Diagnosis and Classification of Diabetes Mellitus2, the Expert Committee on the Diagnosis and Classification of Diabetes Mellitus. Follow-up report on the diagnosis of diabetes mellitus. Diabetes Care 2003; 26:3160-3167.

5. Secretaría de Salud. Encuesta Nacional de Salud y Nutrición 2012, Instituto Nacional de Salud Pública. México [sitio en internet]. [Consultado agosto 28 de 2013]. Disponible en: http://ensanut.insp.mx/ doctos/analiticos/DiabetesMellitus.pdf

6. Secretaría de Salud. Encuesta Nacional de Salud y Nutrición 2012, Resultados por entidad federativa: Hidalgo. Instituto Nacional de Salud Pública. México [sitio en internet]. [Consultado abril 14 de 2015]. Disponible en: http://ensanut.insp.mx/informes/Hidalgo-OCT.pdf.

7. Secretaría de Salud. Manual de Procedimientos Estandarizados para la Vigilancia Epidemiológica de la Diabetes Mellitus Tipo 2. Dirección General de Epidemiología. México. 2012.

8. Rodríguez-Saldaña J, Jonguitud-Falcón A, Clark $C L$, Escorza-Domínguez $A B$, Morales de Teresa $M$, Ortiz-Gress AA. Optimizar la atención sanitaria en México con recursos limitados. Diabetes Voice 2003; 48(3):20-22.

9. Frenk J, González-Pier E, Gómez-Dantés O, Lezana MA, Knaul FM. Reforma integral para mejorar el desempeño del sistema de salud en México. Salud Pública Mex 2007; 49(1):S23-S36.

10. González-Pier E, Gutiérrez-Delgado C, Stevens G, Barraza-Lloréns M, Porras-Condey R, Carvalho N, et al. Definición de prioridades para las intervenciones de salud en el Sistema de Protección Social en Salud de México. Salud Pública Mex 2007; 49(1):S37-S52 
11. Diario Oficial de la Nación. NORMA Oficial Mexicana NOM-015-SSA2-2010, Para la prevención, tratamiento y control de la diabetes mellitus. [sitio en internet]. [Consultado abril 15 de 2015]. Disponible en: http://dof.gob.mx/nota_detalle.php?codigo=5168074\&fecha=23/11/2010

12. Tratamiento de la Diabetes Mellitus tipo 2 en el primer nivel de Atención. México: Instituto Mexicano del Seguro Social. [sitio en internet]. [Consultado abril 15 de 2015]. Disponible en: http://www. cenetec.salud.gob.mx/descargas/gpc/CatalogoMaestro/718_GPC_Tratamiento_de_diabetes_mellitus_ tipo_2_/718GER.pdf

13. Secretaría de Salud. REGLAMENTO de la Ley General de Salud en Materia de Investigación para la Salud. [sitio en internet]. [Consultado julio 28 de 2013]. Disponible en: http://www.salud.gob.mx/ unidades/cdi/nom/compi/rlgsmis.html

14. Epping-Jordan J, Galea G, Tukuitonga C, Beaglehole R. Preventing chronic diseases: taking stepwise action. Lancet 2005; 366:1667-1671.

15. Saydah SH, Fradkin J, Cowie CC. Poor control of risk factors for vascular disease among adults with previously diagnosed diabetes JAMA 2004; 291(3):335-342

16. Villegas-Perrasse A, Abad-Sol B, Faciolince S, Hernández N, Maya C, Parra Lucrecia, et al. El control de la diabetes mellitus y sus complicaciones en Medellín, Colombia, 2001-2003. Rev Panam Salud $2006 ; 20(6): 393-402$.

17. Córdova-Villalobos JA, Barriguete-Meléndez JA, Lara-Esqueda A, Barquera S, Rosas-Peralta $M$, Hernández-Ávila $M$, et al. Las enfermedades crónicas no transmisibles en México: sinopsis. epidemiológica y prevención integral. Salud Pública Mex 2008; 50:419-427.

18. Reid KS, Martin BD, Duerksen F, Nicolle LE, Garrett M, Simonsen JN, et al. Diabetic foot complications in a northern Canadian aboriginal community, Foot Ankle Int 2006; 27(12):1065-1073.

19. Kramer H. Screening for kidney disease in adults with diabetes and prediabetes. Curr Opin Nephrol Hypertens 2005; 14(3):249-255.

20. Górriz Teruel JL, Otero González A. Social and health impact of advanced chronic kidney disease. Nefrologia 2008; 28(3):7-15.

21. Laliberté F, Bookhart BK, Vekeman F, Corral M, Duh MS, Bailey RA, et al. Direct all-cause health care costs associated with chronic kidney disease in patients with diabetes and hypertension: a managed care perspective. J Manag Care Pharm 2009; 15(4):312-322.

22. Barquera S, Campos-Nonato I, Aguilar-Salinas C, López-Ridaura R, Arredondo A, Rivera-Dommarco J. Diabetes in Mexico: cost and management of diabetes and its complications and challenges for health policy. Global Health 2013; 9:3. 